\title{
How low can you go? Detecting style in extremely low resolution images
}

Rachel A. Searston (corresponding author)

School of Psychology, Faculty of Health and Medical Sciences, The University of Adelaide, Australia

Email: rachel.searston@gmail.com

Phone: +61 (0) 447193971

Address: The University of Adelaide, Adelaide, South Australia 5005, Australia

Matthew B. Thompson

School of Psychology and Exercise Science, Murdoch University, Australia

Email: mbthompson@gmail.com

John R. Vokey

Department of Psychology, University of Lethbridge, Canada

Email: vokey@uleth.ca

Luke A. French

School of Psychology, The University of Queensland, Australia

Email: luke.french@uqconnect.edu.au

Jason M. Tangen

School of Psychology, The University of Queensland, Australia

Email: jtangen@psy.uq.edu.au

Word count: 8,726 


\begin{abstract}
Humans can see through the complexity of scenes, faces, and objects by quickly extracting their redundant low-spatial and low-dimensional global properties, or their style. It remains unclear, however, whether semantic coding is necessary, or whether visual stylistic information is sufficient, for people to recognize and discriminate complex images and categories. In two experiments, we systematically reduce the resolution of hundreds of unique paintings, birds, and faces, and test people's ability to discriminate and recognize them. We show that the stylistic information retained at extremely low image resolutions is sufficient for visual recognition of images, and visual discrimination of categories. Averaging over the three domains, people were able to reliably recognize images reduced down to a single pixel, with large differences from chance discriminability across eight different image resolutions. People were also able to discriminate categories substantially above chance with an image resolution as low as $2 \times 2$ pixels. We situate our findings in the context of contemporary computational accounts of visual recognition, and contend that explicit encoding of the local features in the image, or knowledge of the semantic category, is not necessary for recognizing and distinguishing complex visual stimuli.
\end{abstract}

\title{
Public Significance Statement
}

Accurate recognition and discrimination of complex visual stimuli is critical to human decision-making in medicine, forensic science, aviation, security, and defense. This study highlights the sufficiency of redundant low-spatial and low-dimensional information for visual recognition and visual discrimination of three large-scale natural image sets.

Keywords: Visual recognition, visual discrimination, ensemble, gist, perceptual expertise 


\section{How low can you go? Detecting style in extremely low resolution images}

For me, music and life are all about style.

- MiLeS DAVIS.

Humans have a remarkable ability to pick up on redundant visual stylistic information in impoverished images, and under noisy conditions. Radiologists, for example, can detect abnormal mammograms when given a half-second glimpse (Evans, Georgian-Smith, Tambouret, Birdwell, \& Wolfe, 2013; Evans, Haygood, Cooper, Culpan, \& Wolfe, 2016). Fingerprint experts can identify prints that belong to the same finger at a glance, and filtered with artificial noise (Thompson \& Tangen, 2014). They can even detect pairs of prints left by different fingers of the same person (Searston \& Tangen, 2017a), and can search an array of fingerprints for a target more rapidly and accurately than a novice (Searston \& Tangen, 2017b; Searston \& Tangen, 2017c). Looking to more conventional perceptual domains, earlier demonstrations in face recognition revealed that, 'coarse quantized' or reduced resolution versions of a familiar face image (e.g., the Mona Lisa) remained recognizable at just $16 \times 16$ pixels (Harmon \& Julesz, 1973; Morrone, Burr \& Ross, 1983). Torralba (2009) revived this resolution reduction approach and showed that people can identify the semantic category in a variety of indoor (e.g., kitchen, office) and outdoor (e.g., forest, beach) scenes with 81.8\% accuracy when each of the images were $32 \times 32$ color pixels. This same resolution seems to be sufficient for a similar level of image recognition performance (Wolfe \& Kuzmova, 2011). We extend on this body of empirical work here to explore how we accomplish these perceptual feats with so little information at hand.

Prior work that has pushed the limits of human visual recognition with natural images seems to suggest that global properties are sufficient for classification and recognition. 
Indeed, contemporary computational accounts of visual recognition consistently emphasize the importance of the ensemble, the gist, and the spatial envelope for interpreting the contents of natural images, referencing "spatially distributive," "non-spatially localized," or "low frequency" global properties (Brady, Shafer-Skelton \& Alvarez, 2017; Oliva \& Torralba, 2001, Greene \& Oliva, 2009). A rapid and automatic process of extracting coarse-grained global information (e.g., color blobs) is thought to constrain subsequent selective attention and analysis of finer-grained local image properties (e.g., the subtle dimpling that bookends Mona Lisa’s smile; Schyns \& Oliva, 1994; Oliva \& Torralba, 2006). In scene recognition terms, Torralba (2009) describes "gist" as a summary of the semantic content of the scene (i.e., its category label), its spatial layout, and a few objects that compose the scene. In face recognition terms, holistic or "configural" information refers to the spatial relationships between face features (Richler, Mack, Gauthier, \& Palmeri, 2009). Although these descriptions are homing in on the same concept, we think there is inconsistency in the literature about whether references to such global properties are attempting to describe information that is distributed within a particular image, or information that is distributed across images, or a mixture of both. These two different kinds of global information, and their relative necessity for visual recognition, appear to be conflated or unspecified in current theorizing.

We use visual style here to describe the residual redundant information distributed within and across extremely low resolution image sets. A complete exposition of the concept is beyond the reach of this paper, however, we conceive of visual stylistic information as representing the earliest principle components of a given pixel space. Visual style embodies the covariant structure, and not simply the average, of such global properties as low-spatial frequency information, configural information, and low-level dimensions like hue, luminance, 
orientation, and texture. Style can be distinguished from other terms used to describe global information because it does not embody any semantic properties (e.g., category labels), or local features (e.g., high-level spatial information), and can include both spatial (e.g., configuration) and dimensional (e.g., color) information.

The resolution reduction method first applied by Harmon and Julesz (1973) and Morrone et al. (1983), provides a simple but powerful means of testing the sufficiency of global information for performing a range of perceptual tasks. Reducing the resolution of an image or downsampling effectively eliminates the noisiest, least frequent pixels. To the extent that one image shares a pixel-space with other images in a set, reducing their image resolution also has the effect of reducing high-dimensional noise across the entire set-leaving behind the most redundant, low-dimensional category information. The remaining redundant information might be described as a distributed configuration of global features, like hue and luminance, that covary across images. Indeed, Morrone et al. (1983) point out that high spatial frequency information is minimal compared to low spatial frequency information in extremely low "blocked" resolution images (like those produced using a simple nearest neighbor resampling procedure; see Figure 2 for some examples from our image set). By reducing local image features so drastically, and retaining the visual structure across the image set, we can examine the sufficiency of this information for a range of fundamental perceptual tasks: visual object recognition, visual categorization, and as we focus on here, visual discrimination.

Torralba (2009) presented 28 participants with 240 images of indoor and outdoor scenes that were all the same physical size (i.e., all upsampled to $256 \times 256$ pixels) but varied in the number of pixels: ranging from $4 \times 4$ to $128 \times 128$. They used a 12 -alternative forced choice task where participants were asked to classify each image as a member of one of 12 semantic categories (6 indoor and 6 outdoor scenes). Chance level in their experiment was $8.3 \%$ and, as 
a colored image lower bound, $4 \times 4$ pixels was enough to produce a correct categorization rate of $18.4 \%$. Wolfe and Kuzmova (2011) built on this finding by using a two-alternative forced choice image recognition memory task (with an average lag of 32 trials) instead of the categorization task. When accounting for the different chance levels, they found a similar pattern of performance to Torralba (2009) across image resolutions ranging from $8 \times 8$ pixels up to $256 \times 256$ (but did not go any lower than $8 \times 8$ pixels). The fact that people can categorize complex scenes with a resolution as low as $4 \times 4$ pixels, and recognize them with $8 \times 8$ pixels, suggests that they are remarkably sensitive to global features within and distributed across images. Less clear is the basis for this sensitivity: is semantic coding of the image category (the conceptual back-end) necessary for above-chance sensitivity at extremely low resolutions? Is the global information retained in the low resolution images (the perceptual front-end) sufficient in the absence of such semantic coding of the category or any local image features?

We address these questions by probing people's sensitivity to global image (Experiment 1) and category (Experiment 2) information using a resolution reduction methodology similar to prior scene recognition work. However, we add some additional image resolutions and use an adapted experimental design to test just how low people can go without any cues to the semantic category or content of an image. First, in Experiment 1, we extend the work of Wolfe and Kuzmova (2011). Here, we test people's image recognition memory performance using eight image resolution conditions, reducing the images down from $128 \times 128$ to a single pixel. We also use three large sets of images from a variety of different domains (i.e., paintings, birds, and faces) to test the general lower bounds of human visual recognition with extreme image reduction. Wolfe and Kuzmova (2011) suggest that correctly encoding the semantic category - the conceptual back-end-aids picture memory, 
resulting in better performance with higher resolutions, that: "knowing what you are seeing helps you remember it." We test how well people can recognize natural images without necessarily knowing what they are seeing, by reducing the image resolution down to a single pixel and counterbalancing the categories across old and new images. With this design, even if people can recognize an "owl," a "Cubist" painting, or a "female" face from a single colored pixel, or configurations of four, eight, even 16 pixels, the category label would not be diagnostic of whether they had seen that particular patch of pixels before or not, and thus cannot be relied on for recognition.

In Experiment 2, we extend the work of Torralba (2009). Identifying the semantic category of an image below $4 \times 4$ pixels poses quite a challenge. It may be possible, however, to detect the remaining global properties distributed across images at extremely low resolutions using a two-alternative category discrimination task—where participants don't necessarily need to know the semantic categories in order to distinguish their perceptual features. Our guiding hypothesis across the two experiments is that global image features, distributed within and across images, are sufficient for above-chance detection of natural images and categories, even when the semantic content is no longer diagnostic or interpretable. Our preregistered predictions for both experiments are available on the Open Science Framework, along with our experiment code, de-identified data, and analysis script (https://osf.io/thk2a).

\section{Experiment 1: Recognizing Natural Images at Extremely Low Resolutions}

In Experiment 1, we test whether people can recognize natural images based on their most redundant visual features, by gradually reducing their resolution down to one pixel. As noted by Wolfe and Kuzmova (2011), reducing the resolution of images appears to limit people's capacity to rely on conceptual understanding to aid image recognition. But even simple 
biological systems whose cognitive architecture is not likely to support the construction and use of abstract concepts — ants, bees, wasps, pigeons, and fish — can learn to recognize previously travelled routes (Wehner, Boyer, Loertscher, Sommer \& Menzi, 2006), specific artistic styles (Watanabe, 2001; Watanabe et al., 1995; Wu, Moreno, Tangen \& Reinhard, 2013), photographs that contain people and photographs that do not (Aust \& Huber, 2001; Herrnstein \& Loveland, 1964), faces of conspecifics (Sheehan \& Tibbetts, 2011; Siebeck, Parker, Sprenger, Mäthger, Wallis, 2010), abnormal mammograms (Levenson, Krupinski, Navarro, Wasserman, 2015), and four-letter words (Scarf et al., 2016, 2017; see Vokey et al., 2018 for a familiarity-based account of these findings). As such, it seems plausible that people too, may be able to recognize images above chance under extremely low resolution conditions, without knowledge of the original image category or its content.

In this experiment, participants see 24 blocks ( 8 image resolutions $\times 3$ domains) of 24 trials. Within each block of trials, the first 12 consist of a sequence of 12 images presented on a computer screen one after the other, and participants are asked to remember them. The second 12 consist of another 12 images displayed one after the other, and participants are asked to identify whether each image was presented in the original sequence of 12 or not. We examine participants' average discriminability $(A)$, response bias $(B)$, and rate correct (RCS), collapsing across domains (i.e., paintings, birds, faces). Using a metric of discriminability, where .5 is chance and 1 is perfect performance, we based our preregistered predictions on the results obtained by Wolfe and Kuzmova (2011) whose task and stimuli are most similar to this experiment. We predict that participants' discriminability of old and new items would come close to .51 for the one pixel images, to .56 for the $2 \times 2$ pixel images, and .60 for the $4 \times 4$ pixel images, with a further discrimination improvement as the number of pixels in the images increases. 


\section{Method}

We compiled three large sets of images for the two experiments, which we refer to as domains. Each domain consisted of two subcategories: birds (hawks and owls), faces (males and females), and paintings (Cubist and Impressionist). See Figure 1 for a sample of images from each domain and subcategory. To keep the size of the images constant while varying image resolution, all of the images were first downsampled to eight different resolutions $(128 \times 128,64 \times 64,32 \times 32,16 \times 16,8 \times 8,4 \times 4,2 \times 2$, and $1 \times 1)$ and then upsampled to $256 \times 256$.

See Figures 2 and 3 for an example image from each domain and image resolution.
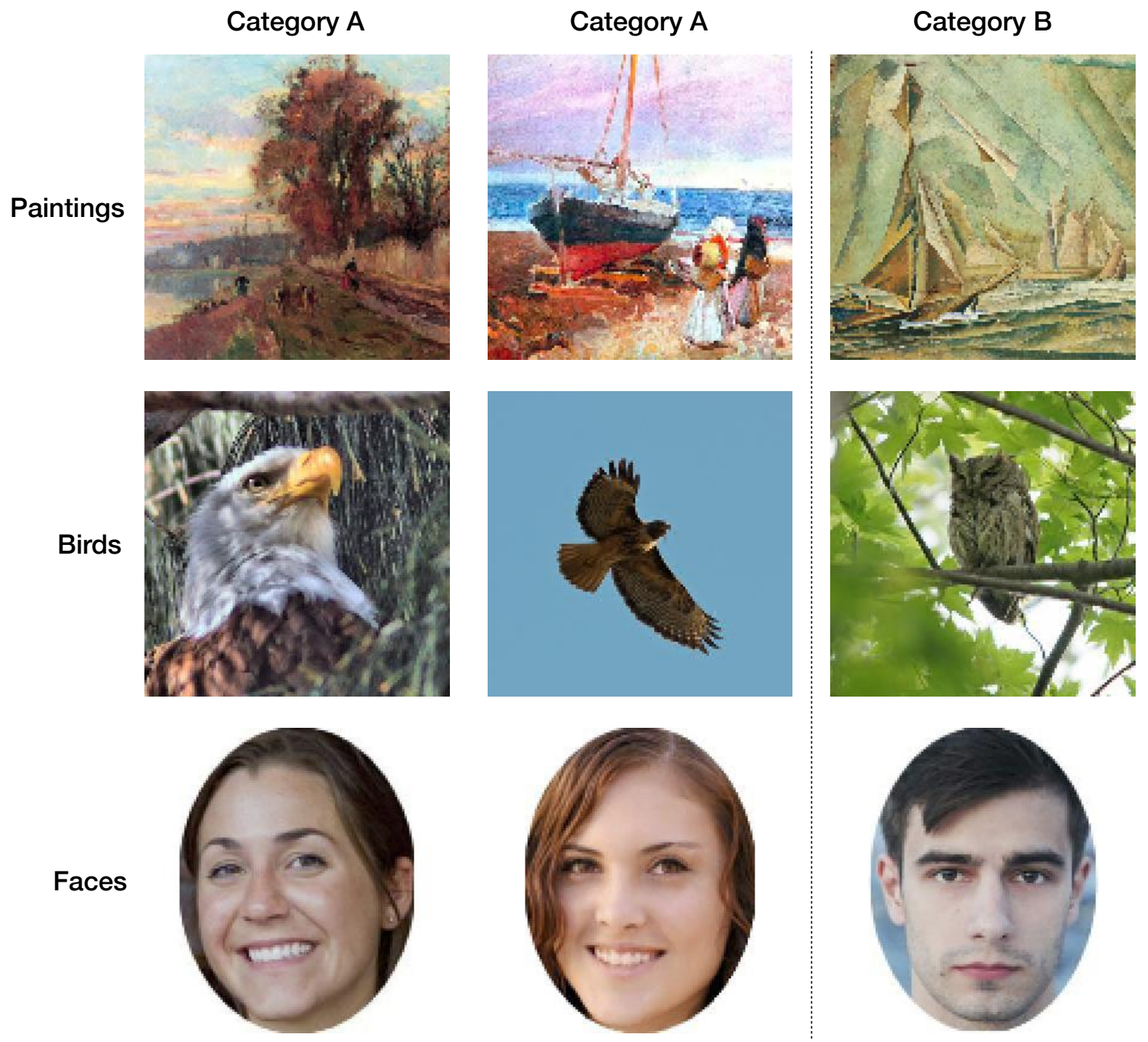
Figure 1. The images to the left of the dotted line are sample "Same" category image-pairs from the three domains at $128 \times 128$ pixels. The images to the right, depict the corresponding "Different" category samples.

\section{Paintings}

The paintings were from a larger art collection created for a previous project. The full collection contains 5,184 paintings, made up of 18 different paintings by 72 different artists, in each of four different artistic styles (Cubism, Impressionism, Realism, and Renaissance; 288 artists in total). We only used the 1,296 Cubist and 1,296 Impressionist paintings here to equate the number of subcategories within the three image sets (2,592 painting in total). The paintings were collected manually from a variety of websites. We cross referenced the paintings to avoid duplicates, and only downloaded images that were larger than $500 \times 500$ pixels. All of the paintings in the art collection were cropped to the centre of the shortest dimension using a 1:1 (square) aspect ratio, and resized (using nearest neighbor scaling) to $256 \times 256$ pixels. Any signatures on the paintings were also removed using the "Content Aware" fill tool in Photoshop.

\section{Birds}

The bird images are a subset of 1,502 extracted from the Cornell Lab of Ornithology's NABirds V1 collection (downloaded from: http://dl.allaboutbirds.org/nabirds). Seven-hundred and fifty-one images, or half of the subset, are natural photographs of birds from the Accipitridae family (e.g., hawks, eagles, kites, harriers, Old World vultures), and the other half are natural photographs of birds from the Strigidae family (owls). The subset contains sixteen different species, with eight species in each family. The species were randomly 
selected to match the number of images between the two families, so both sets of eight species contain $120,103,103,99,73,72,95$, and 86 images per species respectively. We resized each of the images to $256 \times 256$ pixels using a nearest neighbor scaling algorithm and removed any species labels using the "Content Aware" fill tool in Photoshop.

\section{Faces}

The faces are a collection of 1,772 faces images ( 886 female and 886 male) extracted from the 10k US Adult Faces Database (Bainbridge, Isola, \& Oliva, 2013). The entire database contains 10,168 natural face photographs, and we obtained a reduced set of 2,222 images from the database creators along with attribute data (e.g., memorability, attractiveness, happiness, friendliness). We then removed the famous faces from this subset, further reducing it to 2,063 faces ( 886 female, 1,177 male), and extracted the height and width dimensions of the remaining images. All of the images were 256 pixels tall, except one, which we scaled down to match the height of the others. The width of the images ranged from 153 pixels to 157 pixels, and we resized them to $256 \times 256$ pixels. We deleted one image in the male domain that did not match these dimensions. Finally, we randomly sampled 886 male faces from the remaining 1,176 , creating an equal number of male and female images.

\section{Image Resolution}

We generated eight different versions of every image in our three sets, at 1 pixel, $2 \times 2,4 \times 4$, $8 \times 8,16 \times 16,32 \times 32,64 \times 64$, and $128 \times 128$ pixels, using a nearest neighbor resampling procedure in Matlab (see Figures 2 and 3, for an example image from each domain at each of the eight image resolutions). All images were then upsampled to $256 \times 256$ pixels. Although upsampling a set of images using nearest neighbor interpolation produces the blocky looking images illustrated in Figures 1 and 2, the number of pixels in each condition is clear. One intuition about this technique is that the pixel edges or 'blocked' appearance of the images 
introduced at the lower resolutions may serve as high spatial frequency noise masking the lower spatial dimensions. However, Morrone et al. (1983) show that high spatial frequency information is minimal at extremely low resolutions, and that adding high spatial frequency noise can even help unmask low dimensional information — similar to squinting, blurring, shrinking, or looking from a distance.

\section{Participants}

A sample of 50 undergraduate psychology students (33 female, 17 male) from The University of Queensland participated for course credit. Participants' mean age was 20.46 years $(S D=$ 4.73). Ethics approval was received from The University of Queensland Behavioural and Social Sciences Ethical Review Committee (2014001677). Effect sizes were not reported in previous resolution reduction studies, making it difficult to precisely predict magnitude of effects in our experiment ahead of time. Torralba (2009) tested 28 participants who were distributed equally across two groups (greyscale and color images). Wolfe and Kuzmova (2011) tested 73 subjects who were distributed across 11 groups (24 participants were tested in multiple conditions to make 10 participants per group). Considering our use of a withinsubjects design and multiple image sets, we anticipated that our relatively large pre-specified sample of 50 subjects would provide sufficient sensitivity to detect any meaningful differences. Indeed, a sensitivity analysis based on this sample suggests that the smallest effect size we can detect, with $80 \%$ power for our within-subjects Analyses of Variance (ANOVA), accounting for the 576 trials, is $f=.04\left(\eta^{2}=0.002\right.$ or a "small" effect). For the one-sample analyses comparing to chance (i.e., $A=.5$ ), the smallest effect size we can detect with $80 \%$ power is estimated to be $d=.40$ or a "small" to "medium" effect.

\section{Procedure}


Participants completed the experiment (programmed in LiveCode 8.0.0) on a MacBook Air laptop, with a screen resolution of $1440 \times 900$ pixels at $72 \mathrm{dpi}$. They first read an information sheet about the experiment, and then watched an instructional video on the nature of the materials, the image recognition task, and the response scale. Each participant then viewed a series of images, presented one after the other on a solid grey background (R: 209, G: 209, B: 209), to provide contrast and ensure that each image would not blend into the background (i.e., none of the 5,678 images across the three matched that particular shade of grey at 1 pixel). Participants completed 576 trials in total, broken into three (image set: birds, paintings, and faces) $\times$ eight (image resolution: $1,2 \times 2,4 \times 4,8 \times 8,16 \times 16,32 \times 32,64 \times 64$, and $128 \times 128$ pixels) blocks of 24. Each 24-trial block was a "memory" block divided into a learning phase and a test phase. In each learning phase, participants viewed 12 images appearing on screen for 4 seconds at a time, separated by a 500 millisecond interval. In each test phase, participants also viewed 12 images with an old/new response scale appearing on screen. Six of the test images were new and had not been shown previously in the experiment, while the other six were old and had been displayed at some point during the previous learning phase. On a given test trial, participants saw a single image on the screen and rated the extent to which they thought they had seen the image before or not. The image remained on screen until a response was made, but a "speed-up" prompt was delivered if the response time was slower than four seconds. Their responses were recorded by pressing one of 12 buttons on a forced-choice scale with "Old" responses ranging from 1 (Sure Old) to 6 (Unsure Old) and "New" responses ranging from 7 (Unsure New) to 12 (Sure New). The images were sampled equally from the two subcategories in each image set, and presented in a different random order within the learning and test phases of the experiment. Participants were not provided 
with the category labels for any of the image sets, either in the instructional video or during the course of the experiment.

\section{Results}

Of primary interest was the effect of resolution on participants' image recognition memory performance, when averaging over the three image sets or 'domains' (e.g., birds, faces, and paintings). We therefore report analyses of the effect of image resolution on participants' aggregated discriminability ( $A$; see Zhang \& Mueller, 2005 on the correct computation of $A$ instead of $\left.A^{\prime}\right)$, the associated nonparametric measure of response bias $(b)$, and rate correct (RCS; see Vandierendonck, 2018 for an overview of RCS and other speed-accuracy metrics). Our R code and output for all plots, and primary and subsidiary analyses performed for both experiments have been compiled into a single R Markdown HTML document, stored publicly on the Open Science Framework (https://osf.io/thk2a). Separate exploratory analyses of participants' performance with paintings, birds, and faces, in each of the experiments, have also been included in the R Markdown document as supplemental to the main preregistered analyses reported on here.

\section{Discriminability}

In our preregistered analysis plan, we specified that we would compute nonparametric discriminability $\left(A^{\prime}\right)$, because it is intuitive to interpret (e.g., 1 is perfect performance and .5 is chance), and does not assume normally distributed data. We use a corrected formula for $A^{\prime}$ here that was introduced by Zhang \& Mueller (2005) and is denoted as $A$. The new formula can be correctly interpreted as the average of the maximum area and minimum area under the proper Receiver Operating Characteristic (ROC) curve constrained by the hits and false alarms. We first converted participants' confidence ratings into hits and false alarms, and then computed their average discriminability $(A)$ collapsing across domain, for all eight image 
resolutions. We also computed participants' empirical area under the curve (AUC; See Figure 2) to explore whether the pattern of results would change when we accounted for their full range of confidence ratings. Because these additional exploratory analyses yielded the same pattern of results (see the supplemental R Markdown document available on the Open Science Framework for a breakdown of the results and plots of the AUC data across image resolutions: https://osf.io/thk2a), we only report our planned analyses of discriminability using $A$ here.

As can be seen in Figure 2A and Figure 2D, participants' discriminability of Old and New images tended to increase with increasing image resolution: 1 pixel $(A=.66 ; S D=.12)$, $2 \times 2(A=.69 ; S D=.13), 4 \times 4(A=.70 ; S D=.15), 8 \times 8(A=.70 ; S D=.14), 16 \times 16(A=.75 ; S D$ $=.14), 32 \times 32(A=.80 ; S D=.16), 64 \times 64(A=.84 ; S D=.11)$, and $128 \times 128$ pixels $(A=.89$ $S D=.09)$. As planned, a one-way within-subjects ANOVA using participants' $A$ scores revealed a significant main effect of image resolution, $F(7,343)=32.19, p<.001, \eta^{2}{ }_{\mathrm{G}}=.26$ (for a breakdown on using generalized eta-squared as the preferred effect size in an ANOVA context, see Bakeman, 2005). Given that the data distributions for some image resolutions appeared to be skewed, we also conducted a permutation test, permuting participants' $A$ scores $10^{\wedge} 3(1000)$ times, to determine whether a more precise sampling distribution would produce the same result, and it did $(p<.001)$. Polynomial contrasts further revealed a significant linear $(p<.001)$ and quadratic $(p<.001)$ trend to participants' discriminability $(A)$ over image resolutions. This trend was observed even when we accounted for the quadratic increases in the number of pixels across conditions: discriminability $(A)$ increased as the pixel levels went up as integer powers of two (i.e., $2^{0}, 2^{1}, 2^{2}, 2^{3}, 2^{4}, 2^{5}, 2^{6}$, and $2^{7}$ ), with larger increases observed at the higher resolutions (marked by the slight concavity seen in Figure 2A; see the 
supplemental R Markdown document available on the Open Science Framework for a full description of all seven orthogonal contrasts: https://osf.io/thk2a).

We conducted a further finer grained analysis comparing participants' mean discriminability with each image resolution to chance performance $(A=.5)$, and applied corrections to all $p$ values to control the family-wise error rate (Holm, 1979). This analysis revealed that participants performed significantly greater than chance for all image resolution conditions, including the one pixel images, $t(49)=9.36, p<.001, d=1.32$. We report $d$ as defined by Cohen's (1988) original formula (i.e., the difference between our sample mean and chance performance, divided by our sample standard deviation as a substitute for $\sigma$ ) because it deliberately ignores information about the study design and thus allows other researchers to meaningfully compare our effects to different studies. Effect sizes remained large across the image resolution conditions: $2 \times 2$ pixel images, $t(49)=10.32, p<.001, d=1.46 ; 4 \times 4$ pixel images, $t(49)=9.76, p<.001, d=1.38 ; 8 \times 8$ pixel images, $t(49)=9.70, p<.001, d=1.37$; $16 \times 16$ pixel images, $t(49)=12.58, p<.001, d=1.78 ; 32 \times 32$ pixel images, $t(49)=13.10, p<$. $001, d=1.85 ; 64 \times 64$ pixel images, $t(49)=21.60, p<.001, d=3.05 ;$ and $128 \times 128$ pixel images, $t(49)=29.76, p<.001, d=4.21$. Equivalent nonparametric one-sample tests (e.g., Wilcoxon's signed-rank test) produced the same significant results $(p<.001$, applying the Holm correction), across image resolutions (see the supplemental R Markdown file for data analytic code and output: https://osf.io/thk2a $)^{1}$.

\footnotetext{
${ }^{1}$ We report the results of parametric analyses throughout the main text for ease of comparison across studies, however, due to the observed skewness in our data (see the density plots in the supplemental $\mathrm{R}$ Markdown file: https://osf.io/thk2a), we repeated all follow-up one-sample $t$-tests in both experiments with a Wilcoxon's signed-rank test, consistent with our reporting of non-parametric signal detection statistics, and use permutation tests as subsidiary analyses to the ANOVAs.
} 

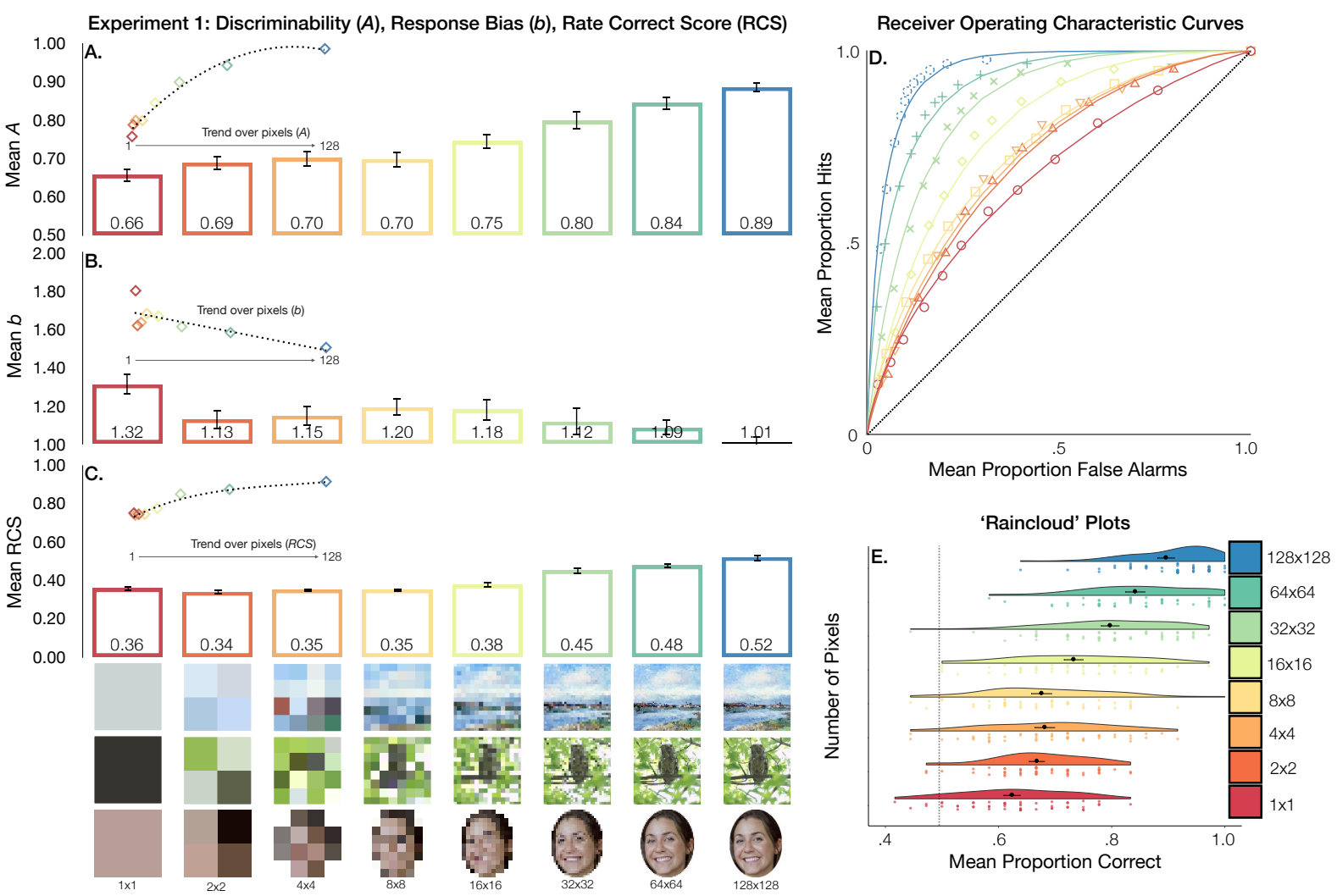

Figure 2. Panels A, B, and C depict participants' mean discriminability $(A)$, response bias $(b)$, and Rate Correct Scores (in seconds) recognition memory task as a function of image resolution ( $x$-axes), along with their polynomial trend over pixels at the top of the three panels. All plots represent the 50 participants' responses, collapsing over the three domains: paintings, birds, and faces. Panel D shows the receiver operating characteristic (ROC) curves for the 8 image resolutions, overlaid with the "best-fitting" curve assuming binormal distributions (the dotted line indicates chance performance). Finally, the raincloud plots in Panel E depict a half violin plot of participants' mean proportion correct scores across the 8 image resolutions overlaid with jittered data points from each individual participant, the mean proportion correct per resolution (the black dot), and standard error of the mean per resolution. 


\section{Response Bias}

We computed participants' average response bias $(b)$ for each image resolution condition from their hits and false alarms, once again averaging over domains (see Figure 2B). A $b$ score of 1 indicates no response bias, with scores greater than 1 indicating a tendency to say, "old" more and scores less than 1 indicating tendency to say, "new" more. Participants in our experiment tended to say, "old" more than "new" $(b=1.15 ; S D=.37)$. This response bias was strongest for the one pixel images (see Figure 2B): 1 pixel $(b=1.32 ; S D=.39), 2 \times 2(b=1.13 ; S D=$. $35), 4 \times 4(b=1.15 ; S D=.36), 8 \times 8(b=1.20 ; S D=.35), 16 \times 16(b=1.18 ; S D=.39), 32 \times 32(b$ $=1.12 ; S D=.53), 64 \times 64(b=1.09 ; S D=.28)$, and $128 \times 128$ pixels $(b=1.01 ; S D=.22) . \mathrm{A}$ one-way within-subjects ANOVA using participants' $b$ scores revealed a significant main effect of image resolution on response bias, $F(7,343)=3.18 p=.003, \eta^{2}{ }_{\mathrm{G}}=.05$. Polynomial contrasts further revealed a significant linear $(p<.001)$ trend to participants' response bias $(b)$ scores over image resolutions. Response bias $(b)$ decreased as the pixel levels went up (see Figure 2B).

We conducted further analyses comparing participants' mean $b$ scores with each image resolution to that expected with no bias $(b=1.00)$. This analysis revealed that participants' response bias towards saying, "old" was significantly greater than no bias for all image resolution conditions (correction applied to all $p$ values). The largest effect was observed with the 1 pixel images, $t(49)=5.72, p<.001, d=.81$. Smaller effect sizes were observed for the other seven image resolutions: $2 \times 2$ pixel images, $t(49)=2.62 p=.012, d=.37 ; 4 \times 4$ pixel images, $t(49)=2.96, p=.005, d=.42 ; 8 \times 8$ pixel images, $t(49)=4.00, p<.001, d=.57$; $16 \times 16$ pixel images, $t(49)=21.25, p<.001, d=.46$; and $64 \times 64$ pixel images, $t(49)=2.25, p$ 
$=.029, d=.32$. No significant response bias was observed for the $32 \times 32$ pixel images, $t(49)$

$=1.63, p=.110$ or $128 \times 128$ pixel images, $t(49)=.43, p=.66$.

\section{Rate Correct}

Finally, we conducted exploratory analyses of participants' Rate Correct Scores (RCSs; see

Figure 2C). Unlike mean response times, RCS provides an indication for participants' speed relative to their accuracy. Compared to other speed-accuracy measures, RCS is also easy to interpret, and can be described here as the number of correct responses per second.

Participants' rate correct remained stable over the lower resolutions, but increased from $32 \times 32$ and up (see Figure 2C): one pixel $(R C S=.36 ; S D=.09), 2 \times 2(R C S=.34 ; S D=.07), 4 \times 4(R C S$ $=.35 ; S D=.07), 8 \times 8(R C S=.35 ; S D=.09), 16 \times 16(R C S=.38 ; S D=.13), 32 \times 32(R C S=.45 ;$ $S D=.13), 64 \times 64(R C S=.48 ; S D=.09)$, and $128 \times 128$ pixels $(R C S=.52 ; S D=.11)$. A oneway within-subjects ANOVA based on participants' mean RCSs also revealed a significant main effect of image resolution, $F(7,343)=47.03, p<.001, \eta^{2}{ }_{\mathrm{G}}=.34$. As with discriminability, polynomial contrasts of the image resolution conditions showed a significant linear $(p<.001)$, quadratic $(p<.001)$, and quartic $(p=.011)$ trend over image resolutions. Rate correct $(R C S)$ increased as the pixel levels went up, and this linear trend was marked by four bends or two step changes at the higher resolutions (see Figure 2C).

\section{Discussion}

We found that people could reliably recognize images above chance (with large effect sizes) at every image resolution, including one pixel. As predicted, people's discriminability of old and new images tended to increase as image resolution increased. On average, participants were also more likely to say that they had seen an image before ("old") than not ("new"), and their rate correct (i.e., correct responses per second) also increased with higher resolutions. 
Looking back in memory, the lower resolution images may appear "older" as a whole because there are fewer distinguishing features between the old and the new images in those conditions. But how are participants remembering $1 \times 1,2 \times 2,4 \times 4$ pixel images reliably? The old and new images were randomly and equally sampled from both categories in all three domains for each memory block. That is, we controlled for the categorical structure of the image sets by counterbalancing the categories across the old and the new images (e.g., in a given painting's memory block: half of the old paintings were randomly sampled from old Cubist artists, and half were randomly sampled from old Impressionist artists, and likewise for the new paintings). Participants may have gleaned the semantic category on any particular image in the experiment, and they may have even noticed that the images tended to be of particular kinds over the course of the experiment. But it was impossible for participants to rely on what they knew about the categorical structure of the image sets to recognize the images, because the categories did not covary systematically with "old" and "new" images. In other words, as a participant in this experiment, knowing that half of the images in a given memory block contained "owls" and half contained "hawks" was not a diagnostic cue to whether you'd seen that image before- because these categories were uncorrelated with the categories "old" and "new."

What information contained within the particular images, then, are participants relying on to recognize them? At the higher resolutions, knowing that an image contains a snowy owl in flight, with flecks of black plumage, on a backdrop of clear blue sky, may help to recognize it as "old" or "new," by virtue of having multiple possible commonalities with the image represented in memory. At the lower resolutions (one pixel, $2 \times 2$ pixels etc.), however, it seems unlikely that participants know there is a feathered, carnivorous bird in the image, and it is therefore unlikely that they are relying on such semantically meaningful content to 
recognize the image. We propose, instead, that participants are relying on the global configural and low-dimensional properties distributed across the image at extremely low resolutions. That is, participants are probably recognizing the "blocky" patterns present at extremely low resolutions, without knowing the semantic category, and without recognizing any meaningful local content in the images. If this explanation holds, the low resolution results provide evidence that we don't need to know what we are seeing in order to remember it (Wolfe and Kuzmova, 2011). The fact that people can remember images that have been down-sampled to a single pixel, even $2 \times 2$ or $4 \times 4$ pixels, reliably above chance, suggests that global features distributed across the image can be sufficient for recognition.

\section{Experiment 2: Discriminating Natural Images at Extremely Low Resolutions}

In Experiment 2, we move from an image recognition task to a discrimination task to probe the sufficiency of global features distributed across images for discriminating categories rather than images. Here, we show people two images on screen, side-by-side. Their task is to decide whether these two images are from the same category or two different categories. We test how well 50 undergraduate psychology students can discriminate categories across the same three domains (paintings, birds, and faces) and eight image resolutions $(128 \times 128$, $64 \times 64,32 \times 32,16 \times 16,8 \times 8,4 \times 4,2 \times 2$, and 1 pixel) introduced in Experiment 1 . Again, we examine participants' discriminability $(A)$, response bias, and rate correct, collapsing over domain. Using a metric of discriminability, where .5 is chance and 1 is perfect performance, we based our preregistered predictions on the results obtained by Torralba (2009) whose task and stimuli are most similar to this experiment. We predict a discriminability of .54 for the 1 pixel images, .60 for the $2 \times 2$ pixel images, and .65 for the $4 \times 4$ pixel images, with a further discrimination improvement as the number of pixels in the images increases. 


\section{Method}

\section{Participants}

A separate pre-specified sample of 50 undergraduate psychology students (34 female, 16 male) from The University of Queensland participated for course credit. Participants' mean age was 19.86 years $(S D=3.51)$. A sensitivity analysis based on this sample suggests that the smallest effect size we can detect with $80 \%$ power, accounting for the 768 trials and withinsubjects design, is $f=.037\left(\eta^{2}=0.001\right.$ or a "small" effect). Sensitivity for the one-sample analyses is identical to Experiment 1.

\section{Procedure}

The stimuli and procedure were identical to Experiment 1, with the exception of the task. Participants were asked to rate the extent to which they thought the two images on each trial were from the same category, or two different categories. They indicated their response on a 12-point, forced-choice rating scale, with buttons ranging from 1 (Sure Different) to 6 (Unsure Different) for the "Different" responses and buttons ranging from 7 (Unsure Same) to 12 (Sure Same). After they pressed one of these 12 buttons, the two images disappeared and were replaced 500 milliseconds later with two new images to be rated. The two images remained on screen until a response was made, but a "speed-up" prompt was delivered if they took longer than four seconds to respond.

Each participant continued this process for 768 trials consisting of 256 pairs of bird images, 256 pairs of paintings, and 256 face pairs, presented as three consecutive blocks in a different random order for each participant. Each 256-trial domain block includes eight smaller blocks of 32 image pairs at each of the eight image resolutions. Each 32-trial resolution block is also presented in a different random order within each domain, and to each participant. The 32 trial blocks are further subdivided into half matching and half 
mismatching image pairs. For example, the correct response on a trial displaying two different Impressionist paintings would be, "same." But the correct response on a trial displaying an Impressionist and Cubist painting would be, "different." Matching and mismatching trials are presented in a different random order within each block of 32, and the images are sampled equally from both subcategories in each domain across all conditions (e.g., Cubist and Impressionist, hawk and owl, male and female). As in Experiment 1, participants were not provided with the category labels for any of the image sets at any point, to minimise reliance on higher-level semantic knowledge. We opted for the label-free visual discrimination task as we were interested in capturing participants' perceptual sensitivity, in its purest form, to the similarities and differences in the image sets at reduced resolutions. For clarity of instruction, participants were asked to judge whether the two images are from the same category or two different categories, but they could theoretically perform the task without knowing the names of the categories they are discriminating.

\section{Results}

As in Experiment 1, we report analyses of the effect of image resolution on participants' aggregated nonparametric discriminability $(A)$, the associated nonparametric measure of response bias (b), and Rate Correct Scores (RCS; number of correct response per second). Again, exploratory analyses of participants' AUC data are not reported as they revealed the same pattern of results as our primary planned analyses of nonparametric discriminability $(A)$. 

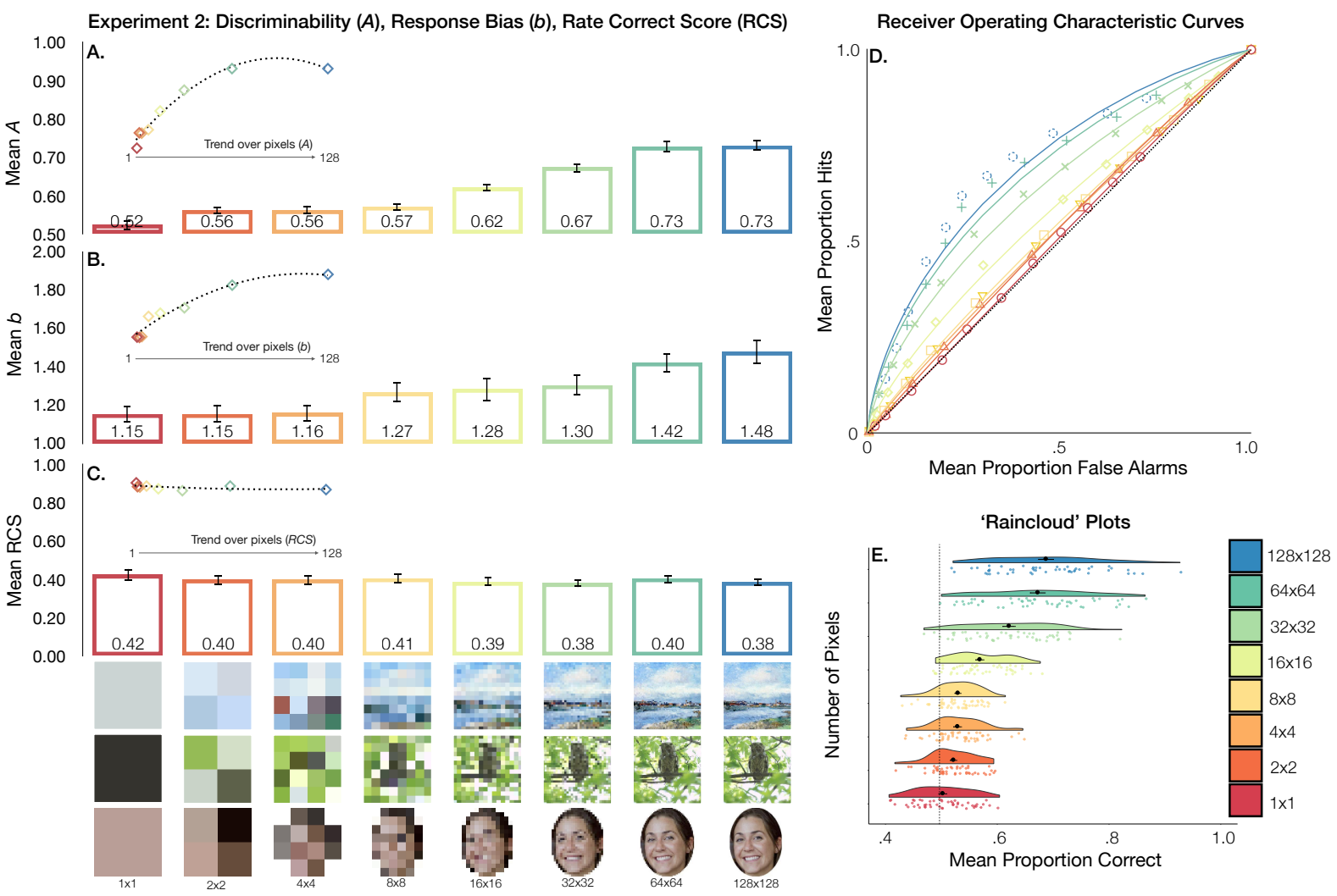

Figure 3. Panels A, B, and C depict participants' mean discriminability $(A)$, response bias $(b)$, and Rate Correct Scores (in seconds) discrimination task as a function of image resolution ( $x$ axes), along with their polynomial trend over pixels overhead. All plots represent the 50 participants' responses, collapsing over the three domains: paintings, birds, and faces. Panel D shows the receiver operating characteristic (ROC) curves for the 8 image resolutions, overlaid with the "best-fitting" curve assuming binormal distributions (the dotted line indicates chance performance). As in Experiment 1, the raincloud plots in Panel E depict a half violin plot of participants' mean proportion correct scores across the 8 image resolutions, overlaid with jittered individual data points, the mean proportion correct per resolution (the black dot), and standard error of the mean per resolution. 


\section{Discriminability}

As illustrated in Figure 3A (see also Figure 3D), participants' discriminability tended to increase with increasing image resolution in a similar fashion to the predicted pattern: one $\operatorname{pixel}(A=.53 ; S D=.09), 2 \times 2(A=.56 ; S D=.07), 4 \times 4(A=.56 ; S D=.08), 8 \times 8(A=.57 ; S D$ $=.07), 16 \times 16(A=.62 ; S D=.07), 32 \times 32(A=.67 ; S D=.08), 64 \times 64(A=.73 ; S D=.10)$, and $128 \times 128$ pixels $(A=.73 ; S D=.10)$. A one-way within-subjects ANOVA using participants' $A$ scores also revealed a significant main effect of image resolution, $F(7,343)=49.84, p<.001$, $\eta^{2}=.44$. Polynomial contrasts of the image resolution conditions revealed a significant linear $(p<.001)$ and quadratic $(p<.001)$ increase in discriminability $(A)$ with increases in the number of pixels. Discriminability $(A)$ increased as the pixel levels went up, and this trend is further characterized by larger increases at higher resolutions (see Figure 3A).

Further one-sample analyses (applying the Holm correction to all $p$ values), revealed that participants' discriminability was reliably greater than chance for all image resolution conditions, except the one pixel images, $t(49)=1.85, p=.070, d=.26$. Effect sizes were large and tended to increase across the remaining seven image resolutions: $2 \times 2$ pixel images, $t(49)$ $=5.98, p<.001, d=.85 ; 4 \times 4$ pixel images, $t(49)=5.60, p<.001, d=.79 ; 8 \times 8$ pixel images, $t(49)=6.85, p<.001, d=.97 ; 16 \times 16$ pixel images, $t(49)=12.49, p<.001, d=1.77 ; 32 \times 32$ pixel images, $t(49)=14.95, p<.001, d=2.12 ; 64 \times 64$ pixel images, $t(49)=15.83, p<.001, d$ $=2.24$; and $128 \times 128$ pixel images, $t(49)=15.60, p<.001, d=2.21$. Discriminability $(A)$ was significantly greater than chance $(p<.001)$ for all image resolutions, including the 1 pixel images $(p=.033)$, using the Wilcoxon signed-rank test and Holm correction (see the supplemental R Markdown file for all data analysis code and output: https://osf.io/thk2a). 


\section{Response Bias}

In Experiment 2, participants tended to say, "different" more than "same" (Mean $=1.28 ; S D=$ .38). This response bias also increased as image resolution increased (opposite to what we predicted): 1 pixel $($ Mean $b=1.15 ; S D=.29), 2 \times 2($ Mean $b=1.15 ; S D=.32), 4 \times 4($ Mean $b=$ $1.16 ; S D=.31), 8 \times 8($ Mean $b=1.27 ; S D=.36), 16 \times 16($ Mean $b=1.28 ; S D=.42), 32 \times 32$ $($ Mean $b=1.30 ; S D=.37), 64 \times 64($ Mean $b=1.42 ; S D=.36)$, and $128 \times 128$ pixels $($ Mean $b=$ 1.48; $S D=.44)$. A one-way within-subjects ANOVA using participants' aggregated $b$ scores revealed a significant main effect of image resolution on their response bias, $F(7,343)=6.66$, $p<.001, \eta^{2}{ }_{\mathrm{G}}=.09$. Similar to participants' discriminability, polynomial contrasts showed a significant linear $(p<.001)$ and quadratic $(p=.040)$ increase in participants' response bias $(b)$ with increasing pixel levels.

Further analyses revealed that participants' response bias towards saying, "different" was significantly greater than no bias for all image resolution conditions, including the 1 pixel images, $t(49)=3.65, p<.001, d=.52$. Effect sizes tended to increase across the eight image resolutions: $2 \times 2$ pixel images, $t(49)=3.39, p=.001, d=.48 ; 4 \times 4$ pixel images, $t(49)=3.67$, $p<.001, d=.52 ; 8 \times 8$ pixel images, $t(49)=5.19, p<.001, d=.73 ; 16 \times 16$ pixel images, $t(49)$ $=4.72, p<.001, d=.67 ; 32 \times 32$ pixel images, $t(49)=5.80, p<.001, d=.82 ; 64 \times 64$ pixel images, $t(49)=8.19, p<.001, d=1.16$; and $128 \times 128$ pixel images, $t(49)=7.64, p<.001, d=$ 1.08 .

\section{Rate Correct}

Participants' mean rate correct remained fairly stable across image resolutions, unlike Experiment 1 (see Figure 3C): 1 pixel $(R C S=.42 ; S D=.24), 2 \times 2(R C S=.40 ; S D=.18), 4 \times 4$ $(R C S=.40 ; S D=.18), 8 \times 8(R C S=.40 ; S D=.18), 16 \times 16(R C S=39 ; S D=.16), 32 \times 32(R C S$ 
$=38 ; S D=.13), 64 \times 64(R C S=.40 ; S D=.16)$, and $128 \times 128$ pixels $(R C S=.39 ; S D=.12) . \mathrm{A}$ one-way within-subjects ANOVA using participants' mean RCSs showed no significant main effect of image resolution, $F(7,343)=.99, p=.442, \eta^{2}{ }_{\mathrm{G}}=.01$, and no significant linear $(p=$. $473)$ or quadratic $(p=.712)$ trend in rate correct with quadratic increases in pixel level.

\section{Discussion}

We found that people could reliably discriminate image categories above chance at image resolutions down to $2 \times 2$ pixels, and less reliably with one pixel. As predicted, people's discriminability tended to increase as image resolution increased. Participants' response bias also tended to increase as image resolution increased, and they tended to say, "different" more often than "same" at all resolutions. There was no evidence for a difference in rate correct across image resolution conditions.

The benefit of randomizing the resolution blocks was in negating the possibility that any observed increases in discriminability with increasing image resolution was a result of participants learning from category exemplars presented earlier in the experiment. Had we increased the resolution blocks incrementally over the experiment, for instance, the observed increased performance would be perfectly confounded with the number of prior category exemplars participants had seen in the experiment. The drawback of randomizing the resolution blocks is that some participants see some higher resolution blocks before the lower resolution blocks, purely based on a random generation process. And participants' exposure to high resolution category exemplars early in the experiment may be inflating their discriminability of the lower resolutions.

If learning in the higher resolution blocks is leaking over into the lower resolution blocks, we would expect to see correlated discriminability across the different blocks. To test this possibility, we correlated participants' discriminability $(A)$ scores in each resolution block 
and with other resolution block. Participants' discriminability was highly correlated among the three highest resolution blocks: $32 \times 32$ and $64 \times 64(r=.638, p<.001), 32 \times 32$ and $128 \times 128(r=.515, p<.001), 64 \times 64$ and $128 \times 128(r=.588, p<.001)$. We also found a moderate correlation between the $2 \times 2$ pixel block and the $16 \times 16$ block $(r=.364, p=.009)$. But discriminability in the $1 \times 1$ pixel block was not correlated at all with discriminability in any other block (relating to the $2 \times 2$ pixel block and up: $r=-.092, r=-.084, r=.051, r=.013$, $r=-.023, r=-.060, r=-.020)$. Discriminability in the $2 \times 2$ pixel block was also not correlated with any other block, except $16 \times 16$ pixels (correlations with the $4 \times 4$ pixel block and up: $r=$. $094, r=.096, r=.364, r=-.049, r=-.042, r=.143)$. Nor was discriminability in the $4 \times 4$ pixel block correlated with any other (correlations with the $8 \times 8$ pixel block and up: $r=.159, r$ $=.131, r=.051, r=-.010, r=.115)$. And discriminability in the $8 \times 8$ pixel block (correlations with the $16 \times 16$ pixel block and up: $r=.113, r=.087, r=.126, r=.252$ ), was not correlated with the $16 \times 16$ pixel block (correlations with the $32 \times 32$ pixel block and up: $r=-.120, r=$. $018, r=.126, r=.081)$,or any of the higher resolutions.

These correlational data show that discriminability at the lower resolutions is not predicted by discriminability at the other resolutions. Apart from the three highest resolutions, discriminability in each block is independent of the other blocks. That is, any familiarity gained with the higher resolution exemplars does not predict performance at the lower resolutions. Prior knowledge of the category exemplars is, therefore, not an adequate explanation for why participants can discriminate categories at extremely low image resolutions. Participants' Rate Correct Scores also didn’t vary significantly across image resolutions in the discrimination task, indicating that the effect of image resolution on discriminability cannot be accounted for by participants emphasising accuracy over speed to a 
greater extent given more information. One plausible explanation for the lack of an effect on rate correct, is that the increasing discriminability with increasing image resolution was offset by increasing complexity in the images - they were slower to respond in the higher image resolution conditions, even though they were more accurate.

With image resolutions as low as $2 \times 2$ or $4 \times 4$ pixels the once nameable local features within the images are no longer present, and thus cannot be used as the basis for discrimination. All that remains is a few patches of color that represent the most redundant information within and across the images in each of the categories. The hawks and owls, for example, appear as different shades of green and brown, the male and female faces as shades of beige. Even as the image resolution increases, the local features - a fine-brushed sailboat, a leafy branch, a flourishing pair of eyebrows - remain obscure.

These findings suggest that people are sensitive to visual information that is distributed across images as well as within images, even when local information is sparse. Although semantic coding at extremely low image resolutions seems unlikely, we speculate that the increase in discriminability at $16 \times 16$ pixels, as indicated by the significant quadratic trend, may mark a point at which participants start to take advantage of semantic coding in their decision process. Similarly, the change in participants' tendency to say, "different" more with the higher image resolutions, might be explained by an increase in the identification of distinguishing objects and features that are local to the images. These post-hoc observations could be tested in future experiments by gauging the point at which participants tend to recognize category labels and objects in an image with gradually increasing image resolution. An interesting prediction based on this interpretation of our results is that semantic coding may influence the decision process at lower and lower image resolutions with the accumulation of experience in a domain, as participants develop visual expertise with natural 
categories (e.g., faces, birds, medical images, fingerprints; Mackenzie, Dodd, Tomarken, \& Gauthier, 2018).

\section{General Discussion}

In two experiments we show that the global stylistic information retained at extremely low image resolutions is sufficient for recognizing images and discriminating categories with above-chance accuracy across three domains - paintings, birds, and faces. We averaged participants' performance over the three large-scale natural image sets and used a random image sampling procedure for each participant and block of trials. We, therefore, expect our findings to generalize well to other natural image sets that have different categorical structures. We distinguish between the style of an image (e.g., a single Monet painting) and the style of a category (e.g., a set of Impressionist paintings) here, because their relative importance appears to depend on the visual task at hand.

Experiment 1, for instance, points to the importance of stylistic information distributed across an image for visual recognition. People were able to reliably recognize natural images reduced down to $4 \times 4$ pixels, $2 \times 2$ pixels, and even a single pixel without any meaningful local objects in the image to rely on. The old and new images were randomly sampled from each of the broader categories in the set, such that their global structure could not be used to aid image recognition. At extremely low resolutions, the images also contain little to no conceptually meaningful local features reminiscent of the original, full-sized images. With a single colored pixel, $2 \times 2$ pixels, or $4 \times 4$ pixels, it seems unlikely that participants are encoding any semantic properties of the original image in order to correctly recognize it. Thus, although an increased ability to perceive meaningful objects and categories in images may help people to remember them (Wolfe \& Kuzmova, 2011), our findings suggest that one need not know to remember. 
Experiment 2, on the other hand, points to the importance of stylistic information distributed across images for discriminating between different visual categories. Participants were able to reliably discriminate images with extremely low resolutions, such as discriminating between a painting by Monet and a painting by Picasso when given only four pixels to go on. Again, at these extremely low resolutions, there are no original local features in the image for people to rely on in order to make a discrimination. As with Herrnstein and Loveland's (1964) pigeons, people appear to show sensitivity to the covariant structure that is distributed across members of a category, in the absence of knowing about their semantic content.

Our notion of style in the image recognition memory task refers to the distributed visual information within the image. No single feature or visual cue (e.g., hue, luminance, orientation) embodies the image's style, but when you look across several features or cues in the image, its style begins to take shape. Extending this description to the discrimination task in Experiment 2, the style of the categories being distinguished cannot be defined by any particular feature or visual cue, or even a set of features within a particular image. Instead, performance on the task depends on a series of features and visual cues that covary across images. An image or category's style is fuzzy, ill-defined and not necessarily tied to any semantic description of its features. Indeed, the style of an image or visual category is analogous to Wittgenstein's (1953) description of family resemblance when describing the covariation of features among the varied instances of "games"- board games and ball games have some commonalities, but also many differences, and it is only when you look across several instances that a resemblance emerges. How people perform on similar tasks, with the within-image and across-image global properties of the stimuli pitted against one another, 
could be tested in future studies, and might help elicit a model of how different kinds of global properties are used in visual cognition.

Previous work has shown that categorization and recognition of scenes decreases as image resolution decreases, remaining above chance at $8 \times 8$ pixels (Torralba, 2009; Wolfe $\&$ Kuzmova, 2011). These findings are interpreted to suggest that the representation of a scene used for recognition memory may be similar to the representation used for categorization, and that images are better remembered when they are meaningful (Wolfe, Horowitz, \& Michod, 2007). These findings, and ours, are consistent with computational vision accounts that propose a coarse-to-fine process by which ensemble or gist-like "blobs" of information are coded first and then used to guide subsequent analyses and recognition of finer details (Schyns \& Oliva, 1994; Oliva \& Torralba, 2006; Torralba \& Oliva, 2003). Unlike gist, however, which is thought to embody a scene's semantic description, spatial layout, and a few select objects that compose the scene (Torralba, 2009), our data provide evidence that explicit encoding of the semantic category or any local features in an image is not necessary for recognizing natural images or distinguishing among natural categories. Visual recognition and discrimination under extremely low resolutions appear to be more about style. 


\section{Disclosures}

\section{Preregistration}

Experiment 1 and Experiment 2 were preregistered on the Open Science Framework (Project link: https://osf.io/thk2a/).

\section{Subjects}

The reported studies were cleared in accordance with the ethical review processes of The University of Queensland and within the guidelines of the National Statement on Ethical Conduct in Human Research (Ethical clearance number: 2010000106). The research was conducted in accordance with the Declaration of Helsinki.

\section{Data, materials, measures, and online resources}

Our experiment code, summary data, plots, and analysis script are publicly accessible online (Project link: https://osf.io/thk2a/). We report how we determined our sample size, all data exclusions, all manipulations, and all measures in the study (see Simmons, Nelson, \& Simonsohn, 2011).

\section{Author Contributions}

RAS, JMT, MBT, and JRV jointly generated the idea for the experiments. JMT resized the images and generated the low resolution images. JMT, RAS, and MBT designed the experiments. RAS wrote the code for displaying the experiments to participants, and wrote the code for all data analyses. JRV had significant input on the data analytic approach taken, and MBT and JMT reproduced the output. LF contributed to the design of Experiment 2 and collection of data and materials as a part of the requirements for an Honours degree in psychology. RAS drafted the manuscript and generated the figures, and all authors provided critical revisions. All authors approved the final submitted version of the manuscript. 


\section{Acknowledgments}

This research was supported by grants from the Australian Research Council (ARC) to J. M. Tangen (DP0985830 and LP120100063), a University of Melbourne McKenzie Postdoctoral Fellowship to R. A. Searston, and a University of Queensland Postdoctoral Fellowship to M. B. Thompson. The authors declare that they have no conflicts of interest with respect to their authorship or the publication of this article. The bird images used were derived from data provided by the Cornell Lab of Ornithology, with thanks to photographers and contributors of crowdsourced data at http://merlin.allaboutbirds.org. This material is based upon work supported by the National Science Foundation under Grant No. 1010818. Yinnam Chan contributed to the design of Experiment 1 and collection of data as a part of the requirements for an Honours degree in psychology. We also thank Brooklyn Corbett for assisting with data collection. We thank Wen Wu, Jessica Marris, Kirsty Kent, and Ryan Metcalfe for assisting with image collection and preparation.

\section{References}

Aust, U., \& Huber, L. (2001). The role of item-and category-specific information in the discrimination of people versus non-people images by pigeons. Animal Learning \& Behavior, 29(2), 107-119. doi: 10.3758/BF03192820

Bainbridge, W. A., Isola, P., \& Oliva, A. (2013). The intrinsic memorability of face images. Journal of Experimental Psychology: General, 142(4), 1323-1334. doi: $10.1037 / \mathrm{a} 0033872$

Bakeman, R. (2005). Recommended effect size statistics for repeated measures designs. Behavior research methods, 37(3), 379-384. doi:10.3758/BF03192707 
Mackenzie, A. S., Dodd, M. D., Tomarken, A. J., Gauthier, I. (2018). How faces (and cars) may become special. Vision Research, S0042-6989(18)30197-4. doi: 10.1016/j.visres. 2017.12.007.

Holm, S. (1979). A simple sequentially rejective multiple test procedure. Scandinavian Journal of Statistics, 6 (2), 65-70. doi: http://www.jstor.org/stable/4615733.

Brady, T. F., Shafer-Skelton, A., \& Alvarez, G. A. (2017). Global ensemble texture representations are critical to rapid scene perception. Journal of Experimental Psychology: Human Perception and Performance, 43(6), 1160-1176. doi: 10.1037/ xhp0000399

Cohen, J. (1988). Statistical Power Analysis for the Behvioral Sciences. Routledge.

Evans, K. K., Georgian-Smith, D., Tambouret, R., Birdwell, R. L., Wolfe, J. M. (2013). The gist of the abnormal: Above-chance medical decision making in the blink of an eye. Psychonomic Bulletin \& Review. doi: 10.3758/s13423-013-0459-3

Evans, K., K., Haygood, T. M., Cooper, J., Culpan, A-M, \& Wolfe, J. M. (2016). A halfsecond glimpse often lets radiologists identify breast cancer cases even when viewing the mammogram of the opposite breast. Proceedings of the National Academy of Sciences, 113(37) 102921-0297; doi: 10.1073/pnas.1606187113

Harmon, L. D. \& Julesz, B. (1973). Masking in visual recognition: Effects of two-dimensional noise. Science, 180, 1194-1197.

Herrnstein, R. J. \& Loveland, D. H. (1964). Complex visual concept in the pigeon. Science, $146,549-551$.

Levenson, R. M., Krupinski, E. A., Navarro, V. M., Wasserman, E. A. (2015). Pigeons (columba livia) as trainable observers of pathology and radiology breast cancer images. PloS ONE, 10(11): e0141357. doi:10.1371/journal.pone.0141357 
Morrone, M. C., Burr, D. \& Ross, J. (1983). Added noise restores recognizability of coarse quantized images. Nature, 305, 226-228.

Oliva, A., \& Torralba, A. (2001). Modeling the Shape of the Scene: A holistic Representation of the Spatial Envelope. International Journal of Computer Vision, 49(3), 145-175.

Torralba, A., \& Oliva, A. (2003). Statistics of natural image categories. Network: Computation in Neural Systems, 14, 391-412.

Oliva, A., \& Torralba, A. (2006). Building the gist of a scene: The role of global image features in recognition. Progress in Brain Research: Visual Perception, 155, 23-36. doi: 10.1016/S0079-6123(06)55002-2

Richler, J. J., Mack, M. L., Gauthier, I., \& Palmeri, T. J. (2009). Holistic processing of faces happens at a glance. Vision Research,49(23), 2856-2861. doi: http://doi.org/10.1016/ j.visres.2009.08.025

Scarf, D., Boy, K., Reinert, A. U., Devine, J., Güntürkün, O., \& Colombo, M. (2016) Orthographic processing in pigeons (Columba livia). Proceedings of the National Academy of Sciences, 113(40):11272-11276.

Scarf, D., Corballis, M. C., Güntürkün, O., Colombo, M. (2017) Do 'literate’ pigeons (Columba livia) show mirror-word generalization? Animal Cognition, 20(5):999-1002.

Schyns, P. G., \& Oliva, A. (1994). From blobs to boundary edges: Evidence for time- and spatial scale-dependent scene recognition. Psychological Science, 5, 195-200.

Searston, R. A., \& Tangen, J. M. (2017a). The style of a stranger: Identification expertise generalizes to coarser level categories. Psychonomic Bulletin \& Review, 24(4), 13241329. doi: 10.3758/s13423-016-1211-6 
Searston, R. A., \& Tangen, J. M. (2017b). Expertise with unfamiliar objects is flexible to changes in task but not changes in class. PLoS ONE, 12(6): e0178403. doi: 10.1371/ journal.pone.0178403

Searston, R. A., \& Tangen, J. M. (2017c). The emergence of perceptual expertise with fingerprints over time. Journal of Applied Research in Memory \& Cognition, 6(4), 442451. doi: 10.1016/j.jarmac.2017.08.006

Searston, R. A., Thompson, M. B., Vokey, J. R., French, L., \& Tangen, J. M. (2018). How low can you go? Detecting style in extremely low resolution images. Supplemental Material: Data Summary, Plots, and Analyses. doi: 10.17605/OSF.IO/BCNKQ

Sheehan, M. J. \& Tibbetts, E. A. (2011). Specialized face learning is associated with individual recognition in paper wasps. Science, 334(6060), 1272-1275. doi: 10.1126/ science. 1211334

Siebeck, U. E., Parker, A. N., Sprenger, D., Mäthgar, L. M., Wallis, G. (2010). A species of reef fish that uses ultraviolet patterns for covert face recognition. Current Biology, 20(5), 407-410. doi:10.1016/j.cub.2009.12.047

Thompson, M. B, \& Tangen, J. M. (2014). The nature of expertise in fingerprint matching: Experts can do a lot with a little. PLoS ONE 9(12): e114759. https://doi.org/10.1371/ journal.pone.0114759

Torralba, A. (2009). How many pixels make an image? Visual Neuroscience, 26, 123-131. doi: $10.1017 / \mathrm{S} 0952523808080930$

Vandierendonck, A. (2018) Further Tests of the Utility of Integrated Speed-Accuracy Measures in Task Switching. Journal of Cognition, 1(1), 8, 1-16, doi: 10.5334/joc.6

Vokey, J. R., Jamieson, R. K., Tangen, J. M., Searston, R. A., \& Allen, S. W. (2018). A visual familiarity account of evidence for orthographic processing in pigeons (Columbia livia): 
A reply to Scarf, Corballis, Güntürkün, and Colombo (2017). Animal Cognition, 21(3), 425-431. doi: 10.1007/s10071-018-1166-2

Watanabe, S. (2001) Van Gogh, Chagall and pigeons: picture discrimination in pigeons and humans. Animal Cognition, 4, 147-151. doi: 10.1007/s100710100112

Watanabe, S., Sakamoto , J., Wakita, M. (1995). Pigeon's discrimination of paintings by Monet and Picasso. Journal of Experimental Analysis of Behaviour, 63, 165-174. doi: 10.1901/jeab.1995.63-165

Wehner, R., Boyer, M., Loertscher, F., Sommer S., \& Menzi, U. (2006). Ant navigation: oneway routes rather than maps. Current Biology, 16(1), 75-79. doi:10.1016/j.cub. 2009.08.015

Wittgenstein, L. (2001) [1953]. Philosophical Investigations. Blackwell Publishing. ISBN $0-631-23127-7$.

Wolfe, J. M., Horowitz, T. S., \& Michod, K. O. (2007). Is visual attention required for robust picture memory? Vision Research, 47, 955-964. doi: 10.1016/j.visres.2006.11.025

Wolfe, J. M., \& Kuzmova, Y. I. (2011). How many pixels make a memory? Picture memory for small pictures. Psychonomic Bulletin \& Review, 18(3), 469-475. doi: 10.3758/ s13423-011-0075-z

Wu, W., Moreno, A. M., Tangen, J. M., \& Reinhard, J. (2013). Honeybees can discriminate between Monet and Picasso paintings. Journal of Comparative Physiology A: Neuroethology, Sensory, Neural, and Behavioral Physiology, 199(1), 45-55. doi: $10.1007 / \mathrm{s} 00359-012-0767-5$

Zhang, J., \& Mueller, S. T. (2005). A note on ROC analysis and non-parametric estimate of sensitivity. Psychometrika, 70, 145-154. doi: 10.1007/s11336-003-1119-8 